\title{
ARTICLE \\ Group II metabotropic glutamate receptor blockade promotes stress resilience in mice
}

\author{
Jaclyn N. Highland (D) ${ }^{1,2}$, Panos Zanos ${ }^{1}$, Polymnia Georgiou ${ }^{1}$ and Todd D. Gould ${ }^{1,2,3,4,5}$
}

\begin{abstract}
Stress is a leading risk factor for the onset and recurrence of major depression. Enhancing stress resilience may be a therapeutic strategy to prevent the development of depression in at-risk populations or its recurrence in depressed patients. Group II metabotropic glutamate receptor $\left(\mathrm{mGlu}_{2 / 3}\right)$ antagonists have been recognized for antidepressant-like actions in preclinical models, but have not been evaluated for prophylactic effects. We assessed the role of $\mathrm{mGlu}_{2 / 3}$ in modulating stress resilience using subtypespecific knockout mice lacking $\mathrm{mGlu}_{2}\left(\mathrm{Grm}^{-1-}\right)$ or $\mathrm{mGlu}_{3}\left(\mathrm{Grm}^{-1-}\right)$, and pharmacological manipulations of $\mathrm{mGlu} 2 / 3$ activity during or prior to the induction and reinstatement of stress-induced behavioral deficits. $\mathrm{Grm}^{-1-}$, but not $\mathrm{Grm}^{-1-}$, mice exhibited reduced forced-swimming test immobility time and were resilient to developing inescapable shock (IES)-induced escape deficits. $\mathrm{Grm}^{-/-}$mice were also resilient to developing corticosterone (CORT)-induced escape deficits and chronic social defeat stressinduced anhedonia. Pharmacological blockade of $\mathrm{mGlu}_{2 / 3}$ with the antagonist LY341495 during stress prevented the development of IES- and CORT-induced escape deficits, while activation with the agonist LY379268 increased susceptibility to escape deficits. Prophylactic treatment with the LY341495, both systemically and via microinjection into the medial prefrontal cortex (mPFC), up to 7 days before IES, prevented both the induction of escape deficits and their reinstatement by brief re-exposure to IES up to 20 days after treatment. Overall, blockade of $\mathrm{mGlu}_{2 / 3}$ enhanced stress resilience and deletion of $\mathrm{mGlu}_{2}$, but not $\mathrm{mGlu}_{3}$, conferred a stressresilient phenotype, indicating that prophylactic treatments reducing $\mathrm{mGlu}_{2}$ activity may protect against stress-induced changes underlying the development or recurrence of stress-induced disorders, including depression.
\end{abstract}

Neuropsychopharmacology (2019) 44:1788-1796; https://doi.org/10.1038/s41386-019-0380-1

\section{INTRODUCTION}

Despite a number of available treatments for major depression, many patients fail to reach remission [1] and the vast majority $(\sim 85 \%)$ of remitted patients experience a recurrence of symptoms [2], highlighting the need for novel, more effective therapies. Stress is a leading risk factor for both the initial onset of depression and the incidence of recurrent episodes (reviewed in $[3,4])$. Thus, enhancing stress resilience may be a therapeutic strategy to prevent the development of depression within predictably at-risk/susceptible populations, or to prevent the recurrence of its symptoms in depressed patients.

While the exact mechanisms underlying stress resilience and susceptibility continue to be elucidated, deficits in excitatory synaptic transmission have been implicated in stress-related disorders including depression. Stress has been reported to weaken glutamatergic synapses in mood-regulating brain regions including the hippocampus and medial prefrontal cortex (mPFC) [5-7]. In rodents, this effect is observed concurrently with behavioral changes inferred to model aspects of depression and reversed by antidepressant treatments [5-7]. In addition, activation of glutamatergic projections from the mPFC to the dorsal raphe (DRN) prevents the development of such maladaptive stress-induced behaviors altogether [8]. Thus, agents that activate or enhance excitatory neurotransmission and increase synaptic strength at these synapses are predicted to increase stress resilience.

A primary role of type II metabotropic glutamate receptors $\left(\mathrm{mGlu}_{2}\right.$ and $\left.\mathrm{mGlu}_{3}\right)$ is their autoreceptor function (reviewed in [9]), whereby activation of $\mathrm{mGlu}_{2 / 3}$ inhibits glutamate release mechanisms, and conversely, blockade of $\mathrm{mGlu}_{2 / 3}$ enhances glutamatergic neurotransmission by relieving this inhibition [10, 11]. This autoreceptor function has been primarily attributed to $\mathrm{mGlu}_{2}$, which is predominantly localized near presynaptic terminals [1214] (whereas $\mathrm{mGlu}_{3}$ is primarily expressed postsynaptically on neurons and on glia [13-15]. Thus, we hypothesized that decreased activity of $\mathrm{mGlu}_{2}$ (predicted to enhance glutamatergic neurotransmission) would enhance stress resilience. While $\mathrm{mGlu}_{2 / 3}$ antagonists have gained attention for their rapid-acting antidepressant actions (i.e., reversal of depression-relevant behaviors) in preclinical studies (e.g., [14, 16-28]), an effect which has been primarily attributed to the $\mathrm{mGlu}_{2}$ subtype $[28,29]$, the ability of these compounds to prevent adverse stress-induced changes has not been extensively studied.

To evaluate the subtype-specific effects of these receptors on the development of stress-induced behavioral changes, we utilized knockout mice lacking either $\mathrm{mGlu}_{2}\left(\mathrm{Grm}^{-1-}\right)$ or $\mathrm{mGlu}_{3}$ $\left(\mathrm{Grm}^{-/-}\right)$. In addition, we implemented pharmacological manipulations of group II mGlu activity with the $\mathrm{mGlu}_{2 / 3}$ antagonist

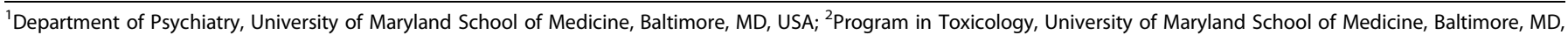

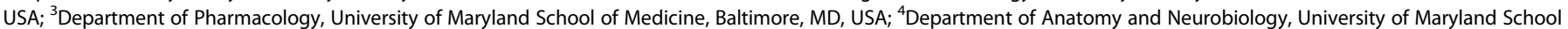
of Medicine, Baltimore, MD, USA and ${ }^{5}$ Veterans Affairs Maryland Health Care System, Baltimore, MD, USA

Correspondence: Todd D. Gould (gouldlab@me.com)

Received: 30 October 2018 Revised: 14 February 2019 Accepted: 25 March 2019

Published online: 2 April 2019 
LY341495 [30] and agonist LY379268 [29]. Specifically, we assessed the effects of bidirectional modulation of $\mathrm{mGlu}_{2 / 3}$ activity on stress resilience in wild-type (WT) mice and evaluated whether pretreatment with LY341495 (either systemically or via intracortical administration to the mPFC) was protective against the onset and reinstatement of maladaptive behaviors in a novel model of recurrent stress susceptibility.

\section{MATERIALS AND METHODS}

Animals

CD-1 mice were obtained from Charles River Laboratories (Raleigh, NC, USA). Knockout mice (Grm2 $2^{-1-}$ and $\mathrm{Grm}^{-1-}, \mathrm{CD}-1$ background (see [31, 32])) and WT controls were provided by Eli Lilly Pharmaceuticals and were maintained at Taconic Biosciences (Hudson, NY, USA). All mice acclimated to the University of Maryland Baltimore animal facility for at least 1 week prior to testing and were housed with a constant 12-h light cycle (lights on at 07:00) and food and water available ad libitum. All testing was performed during the light phase. Experimental mice were 8-12 weeks old at the time of testing and housed 3-5/cage, unless otherwise specified. For CSDS experiments, retired male breeder CD-1 mice (aggressors) were obtained from Charles River Laboratories and were singly housed. All procedures were approved by the University of Maryland Baltimore Animal Care and Use Committee in accordance with the National Institutes of Health Guide for the Care and Use of Laboratory Animals.

\section{Drugs}

LY341495 and LY379268 disodium salts (see Supplementary Materials and Methods for potency and selectivity information) were purchased from Tocris (MN, USA) and dissolved in $0.9 \%$ saline. Corticosterone (CORT) was obtained from Sigma-Aldrich (MO, USA) and initially dissolved in ethanol then diluted 1:10 with $0.9 \%$ saline (final solution contained $10 \%$ ethanol). Systemic injections were administered in a volume of $7.5 \mathrm{ml} / \mathrm{kg}$. LY341495 and LY379268 (free drug dose, $3 \mathrm{mg} / \mathrm{kg}$ ) were administered via intraperitoneal (i.p.) injection. CORT $(10 \mathrm{mg} / \mathrm{kg})$ was injected subcutaneously (s.c.). For intracortical microinjections, saline or LY341495 (0.10 or $1.0 \mathrm{pmol}$ per side) was administered in a volume of $100 \mathrm{nl}$ per side. The doses of LY341495 [21, 25, 30], LY379268 [29], and CORT [33, 34] were selected based upon their reported behavioral effects at similar doses and published pharmacokinetic data.

\section{Cannulation surgery and microinjection}

Cannula implantation and microinjections are described in the Supplementary Materials and Methods. Briefly, bilateral guide cannulas were surgically implanted in the mPFC, and after a recovery period of 6-8 days, saline or LY341495 was infused via an internal cannula (tips positioned $+2.00 \mathrm{~mm}$ anteroposterior, $\pm 0.50 \mathrm{~mm}$ lateral, $-2.50 \mathrm{~mm}$ dorsoventral from bregma). Cannula placements were confirmed after testing; animals with cannulas outside the mPFC (one from each experimental group) were excluded from analysis.

\section{Behavioral tests}

Inescapable shock (IES)-induced escape deficits. The procedure is detailed in the Supplementary Materials and Methods. In brief, mice received 120 inescapable shocks, and $24 \mathrm{~h}$ later were tested over 30 escapable shock trials. The number of escape failures (indicative of "helplessness" or susceptibility) was recorded. Mice were treated with LY341495, LY379268, or saline $35 \mathrm{~min}$ or $24 \mathrm{~h}$ prior to IES.

To assess the recurrence of escape deficits, a modified protocol was implemented; see Supplementary Materials and Methods. Briefly, mice were treated with LY341495 or saline 3 or 7 days prior to an initial IES session (day 4 or 8 ) and tested for escape deficits
24 h later (Test 1, day 5 or 9), as described above. Eleven days later, mice were re-tested using the same procedure to assess recovery (Test 2, day 16 or 21). Twenty-four hours later (day 17 or 21) mice received 20 inescapable shocks and the following day (day 18 or 22), were tested a final time.

Corticosterone (CORT)-induced escape deficits. Acute CORTinduced escape deficits were induced based upon a previously described protocol [33] with modifications; see Supplementary Materials and Methods. In brief, mice received an injection of CORT or vehicle, and $24 \mathrm{~h}$ later, were tested for escape failures as described above. Naive $\mathrm{Grm}_{2}{ }^{-1-}$ and WT mice, and WT mice pretreated with LY341495 or saline $35 \mathrm{~min}$ prior to CORT treatment were tested in separate experiments.

Forced-swimming test (FST). Mice were placed into a clear cylinder $(30 \times 20 \mathrm{~cm}$, height $\times$ diameter $)$ filled to a depth of $15 \mathrm{~cm}$ with water $\left(23 \pm 1{ }^{\circ} \mathrm{C}\right)$ and subjected to an initial 6-min swim session, then re-tested using the same procedure $24 \mathrm{~h}$ later. Immobility (passive floating with only those movements necessary to keep the head above water) time was scored during the final 4 min of each session.

Open-field test (OFT). Mice were placed into an open-field arena (San Diego Instruments, San Diego, CA, USA; $50 \times 50 \times 38 \mathrm{~cm}$; length $\times$ width $\times$ height) for $50 \mathrm{~min}$ and locomotor activity was recorded using an overhead camera. Distance travelled was analyzed using TopScan software (v2.0; CleverSys, Inc., Reston, VA, USA).

Shock sensitivity test. The shock sensitivity test is described in detail in the Supplementary Materials and Methods. Briefly, mice received a series of foot shocks of increasing intensity and a trained observer recorded flinching in response to shock. The lowest intensity at which flinching occurred was determined. In separate experiments, naive $\mathrm{Grm}_{2}{ }^{-1-}$ and WT mice, and WT mice treated with LY341495, LY379268, or saline 90 min before shock onset (timing approximates the midpoint of IES session) were tested.

Passive avoidance test. The passive avoidance test is described in the Supplementary Materials and Methods. In brief, the test consisted of two sessions-training and testing-and was conducted in shuttle boxes consisting of a light and a dark compartment. Mice were placed into the light compartment and the latency to cross into the dark compartment was recorded; upon entry, a single inescapable foot shock was administered (training). Twenty-four hours later, mice were returned to the light compartment and latency to cross was recorded (test). Animals that did not cross after $10 \mathrm{~min}$ were assigned a latency of $600 \mathrm{~s}$. In separate experiments, naive $\mathrm{Grm}_{2}{ }^{-1-}$ and WT mice, and WT mice treated with LY341495, LY379268, or saline $90 \mathrm{~min}$ prior to the training session were tested.

Chronic social defeat stress (CSDS)/sucrose preference test. The CSDS protocol is detailed in the Supplementary Materials and Methods (see Fig. 2d for timeline). Briefly, each day for 10 days, experimental mice underwent 10 -min of physical attack by a larger, aggressive mouse, followed by $24 \mathrm{~h}$ of sensory contact with the aggressor. Anhedonia was subsequently assayed via sucrose preference. We note that CSDS is typically performed using C57BL/ 6 mice for the experimental group with both social interaction and sucrose preference deficits assayed $[35,36]$. However, pilot experiments (data not shown) demonstrated that older, retired breeder CD-1 mice (previously screened for aggressive behaviors) exhibited high levels of aggression toward the younger, experimental CD-1 mice, resulting in the development of sucrose preference deficits, but not social interaction deficits, following 


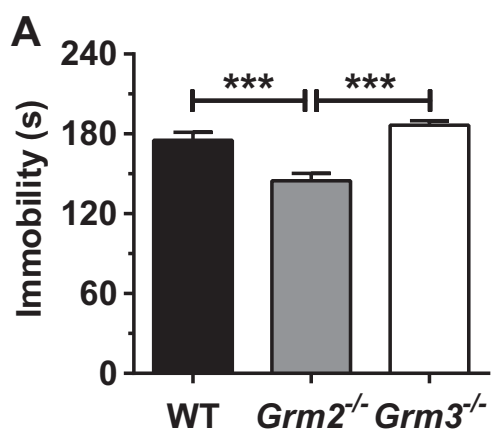

C

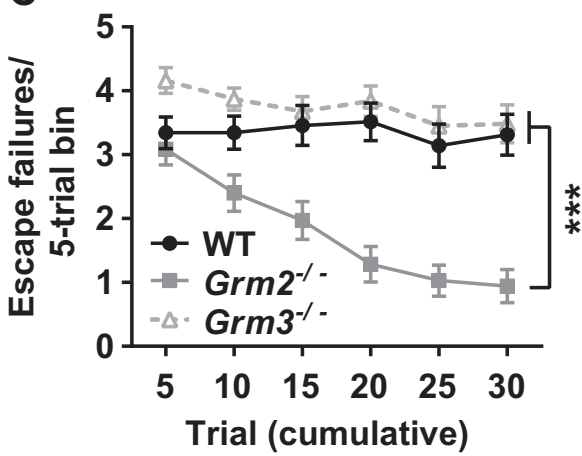

B

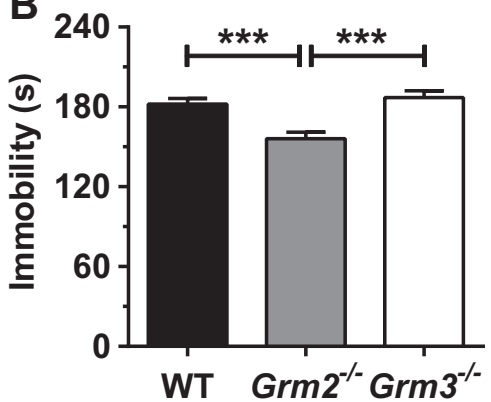

D

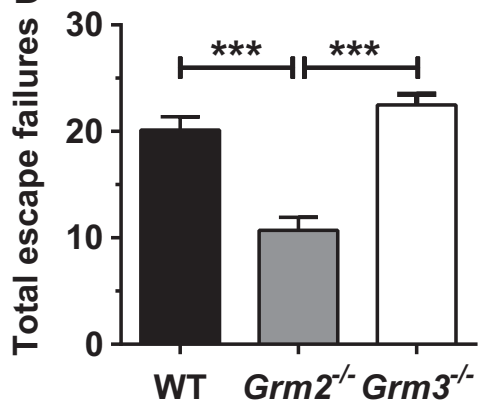

Fig. 1 Mice lacking $\mathrm{mGlu}_{2}$, but not those lacking $\mathrm{mGlu}_{3}$, manifest reduced immobility in the forced-swimming test (FST) and are resilient to inescapable shock-induced escape deficits. Relative to wild-type (WT) controls, mice lacking mGlu $\left(\mathrm{Grm}^{-1-}\right)$, but not those lacking mGlu 3 $\left(\mathrm{Grm}^{-/-}\right)$, present with reduced immobility in (a) an initial FST and (b) a re-test session $24 \mathrm{~h}$ later $(n=23-24 \mathrm{genotype})$. Grm2 ${ }^{-/-}$mice, but not $\mathrm{Grm}^{-1-}$ mice, also manifest fewer $(\mathbf{c}, \mathbf{d})$ inescapable shock-induced escape failures, compared with WT mice (c, failures over time; $\mathbf{d}$, total failures; $n=31-35 /$ group). Data are mean \pm SEM. ${ }^{*} p<0.05,{ }^{* *} p<0.01,{ }^{* *} p<0.001$. See Table S1 for reporting of statistical analyses and sample sizes per sex

CSDS. Therefore, sucrose preference was our primary experimental outcome.

\section{Plasma corticosterone assay}

The procedure is described in the Supplementary Materials and Methods. In brief, trunk blood was collected immediately after IES (as described above) or from unstressed controls. Plasma corticosterone concentrations were measured via radioimmunoassay.

Statistical analysis

All experiments were performed in a randomized and blinded manner. Statistical analyses were performed using GraphPad Prism (v6; GraphPad Software, Inc.), except for three-way ANOVAs, which were performed using Statistica Academic ( $v 13$, TIBCO Software, Inc.). All statistical tests were two-tailed with significance assigned at $p \leq 0.05$. Where no effect of sex was detected, group data were combined for analysis. Where an effect of sex was detected, male and female data were analyzed separately. Tukey post hoc tests were used when ANOVAs reached statistical significance. The specific tests applied, sample sizes, and the statistical effects for each experiment are reported in Table S1. Values are represented as the mean \pm SEM and statistical significance is indicated in figures with asterisks $\left({ }^{*} p \leq 0.05,{ }^{* *} p<\right.$ $\left.0.01,{ }^{* * *} p<0.001\right)$.

\section{RESULTS}

Mice lacking $\mathrm{mGlu}_{2}$, but not those lacking $\mathrm{mGlu}_{3}$, exhibit stress resilience

Compared with WT and $\mathrm{Grm}^{-1-}$ mice, $\mathrm{Grm}^{-1-}$ mice displayed reduced immobility time in an initial FST session (Fig. 1a) and in a re-test session $24 \mathrm{~h}$ later (Fig. $1 \mathrm{~b}$ ). The immobility time of $\mathrm{Grm}^{-/-}$ mice did not differ from that of WT mice (Fig. 1a, b). To further evaluate the subtype-specific effects of $\mathrm{mGlu}_{2 / 3}$ deletion, we assessed the development of IES-induced escape deficits in $\mathrm{Grm}^{-1-}$, Grm3 ${ }^{-1-}$, and WT mice. Twenty-four hours after exposure to IES, Grm2 $2^{-1-}$ mice manifested fewer escape failures (Fig. 1c, d) than either WT controls or $\mathrm{Grm}^{-/-}$mice, while $\mathrm{Grm3}^{-l-}$ mice did not differ from WT controls (Fig. 1c, d). Thus, we focused on further investigating the resilient phenotype of $\mathrm{Grm}^{-/-}$mice. We note that in both tests, there was no interaction with sex.

We evaluated the development of CORT-induced escape deficits (see Fig. 2a for experimental timeline), which are independent of a physical or painful foot shock stressor [33], in $\mathrm{Grm2}^{-1-}$ and WT mice. While an acute injection of CORT induced an increase (relative to vehicle) in the number of escape failures measured $24 \mathrm{~h}$ later in WT mice, Grm2 ${ }^{-/-}$mice lacked these CORTinduced escape deficits (Fig. 2b, c). There was no overall effect of sex. To evaluate whether the effects of $\mathrm{mGlu}_{2}$ deletion extended to chronic stress, we measured the development of CSDS-induced anhedonia in male mice, assayed via sucrose preference (see Fig. 2d for experimental timeline). Following 10-day CSDS, WT mice manifested a significant decrease in sucrose preference, relative to unstressed controls (Fig. 2e). Grm2 ${ }^{-1-}$ mice, however, lacked CSDS-induced sucrose preference deficits, with no significant difference detected between $\mathrm{Grm}^{-1-}$ mice that underwent CSDS and unstressed controls (Fig. 2e). While not systematically evaluated, no robust differences in behavior during CSDS were observed between genotypes. Finally, we measured plasma CORT concentrations in unstressed male mice and immediately following IES. In both WT and $\mathrm{Grm}^{-1-}$ mice, IES induced robust increases in plasma CORT (Fig. 2f), without differences in CORT levels at baseline (i.e., in unstressed mice) or following IES (Fig. 2f). 
A

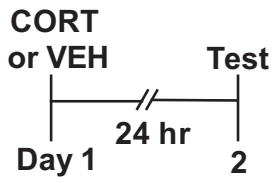

B

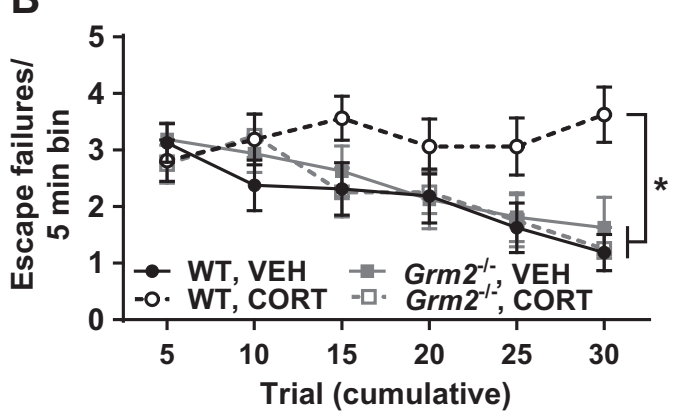

C

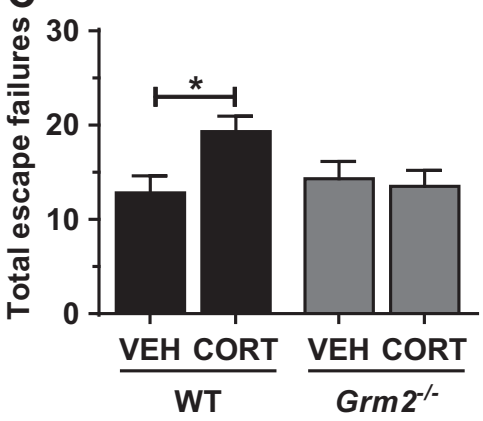

D

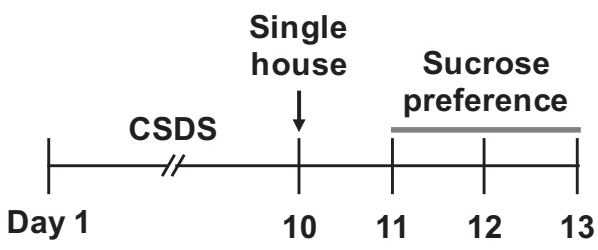

E

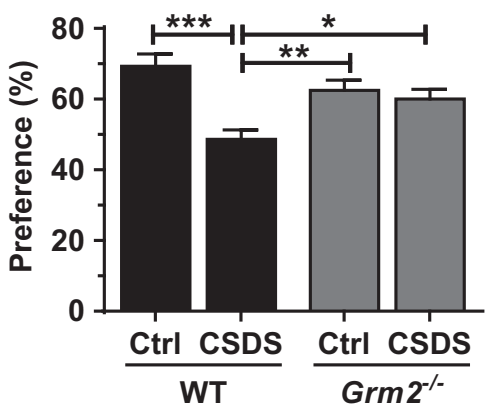

$\mathbf{F}$

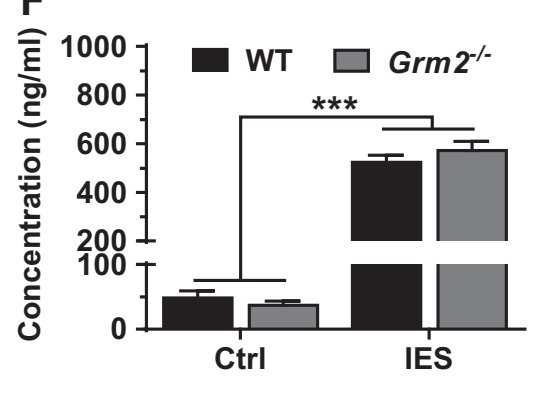

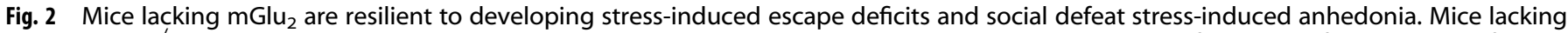
$\mathrm{mGlu}_{2}\left(\mathrm{Grm} 2^{-/-}\right)$are resilient to developing (a-c) acute corticosterone (CORT, $10 \mathrm{mg} / \mathrm{kg}$ )-induced escape deficits (see a for timeline; b, failures over time; c, total failures $n=16 /$ group) and (d, e) chronic social defeat stress (CSDS)-induced sucrose preference deficits (see $\mathbf{d}$ for timeline;

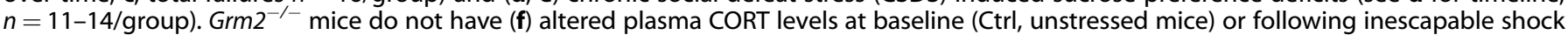

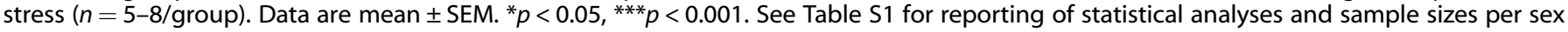

To assess potential locomotor and nociceptive differences that could impact performance in the other behavioral tests, we measured open-field locomotor activity and sensitivity to shock, respectively, in $\mathrm{Grm}^{-1-}$ and WT mice. An overall effect of sex was observed for both outcomes: females manifested slightly higher locomotor activity (Fig. 3a, b) and a lower threshold for shock response (Fig. 3c) than males. However, no differences in locomotion (Fig. 3a, b) or shock response (Fig. 3c) were detected between genotypes for either sex. In addition, we utilized the passive avoidance test to evaluate potential alterations in memory-dependent shock avoidance which could impact IESinduced escape behavior. While an overall effect of sex was observed in the passive avoidance test, with females having higher escape latencies than males, there was no effect of genotype in either sex (Fig. 3d).

Pharmacological modulation of $\mathrm{mGlu}_{2 / 3}$ activity during stress bidirectionally modulates resilience versus susceptibility

We utilized group II mGlu selective ligands to assess whether pharmacological modulation of $\mathrm{mGlu}_{2 / 3}$ activity, at various time points relative to a stressor, alters stress-induced behavioral changes. We first evaluated whether increasing or decreasing $\mathrm{mGlu}_{2 / 3}$ activity during a stressor would promote stress resilience and susceptibility, respectively. Treatment with the $\mathrm{mGlu}_{2 / 3}$ antagonist LY341495, 35-min prior to IES (see Fig. 4a for timeline), robustly attenuated the development of escape deficits measured $24 \mathrm{~h}$ after IES, relative to saline treatment (Fig. 4b, c). This effect was independent of an overall effect of sex. Conversely, treatment with the $\mathrm{mGlu}_{2 / 3}$ agonist LY379268 at the same time point increased escape failures (Fig. 4e, f). We note that this experiment was extended to include a re-test session $24 \mathrm{~h}$ after the initial test (see Fig. $4 \mathrm{~d}$ for timeline), because initial analysis revealed an effect of LY379268 in the final trials of the first test session. To further investigate the stress-protective effects of blocking $\mathrm{mGlu}_{2 / 3}$ during stress, mice were pretreated with
LY341495 or saline $35 \mathrm{~min}$ prior to CORT administration (see Fig. $4 \mathrm{~g}$ for timeline). While CORT induced a robust increase in escape failures measured $24 \mathrm{~h}$ later in saline-treated mice, mice pretreated with LY341495 were resilient to these effects (Fig. 4h, i). This effect was independent of an overall effect of sex. Importantly, neither LY341495 nor LY379268 (administered $90 \mathrm{~min}$ prior to testing; see Fig. $4 \mathrm{j}$ for timeline) altered sensitivity to shock (Fig. 4k) or passive avoidance performance (Fig. 4l, m).

Prophylactic blockade of $\mathrm{mGlu}_{2 / 3}$ prevents the development and recurrence of stress-induced behavioral deficits

To evaluate whether pretreatment with LY341495 was protective against stress exposure after drug clearance from the brain (half-life $\sim 5 \mathrm{~h}$ in the brains of rats [30]), we measured IESinduced escape deficits in male mice treated with LY341495 or saline $24 \mathrm{~h}$ prior to IES (see figure Fig. $5 \mathrm{a}$ for timeline). Compared with saline, pretreatment with LY341495 attenuated the development of IES-induced escape failures measured $24 \mathrm{~h}$ later (Fig. 5b, c). Further, we utilized a novel, repeated IES paradigm (see Figs. 5d, f for timelines) to assess the effects of LY341495 pretreatment on both the development and reinstatement of stress-induced behavioral changes. In this paradigm, vehicletreated mice recover from the initial bout of IES-induced escape deficits within 2 weeks (recovery tested 12 days after IES; Figs. 5e, g, Test 2), but subsequent exposure to a brief IES session ( 20 shocks) reinstates this maladaptive behavior (Figs. 5e, g, Test 3). Treatment with LY341495 3 days before the initial IES session (see Fig. $5 d$ for timeline) prevented both the initial development of IES-induced escape deficits (Fig. 5e, Test 1) and their reinstatement by subsequent exposure to a shorter IES session 16 days after treatment (Fig. 5e, Test 3), without altering recovery (Fig. 5e, Test 2). Treatment with LY341495 7 days before the initial IES session and 20 days before re-exposure to an abbreviated IES session (see Fig. $5 f$ for timeline) also prevented the development (Fig. $5 \mathrm{~g}$, Test 1) and reinstatement 

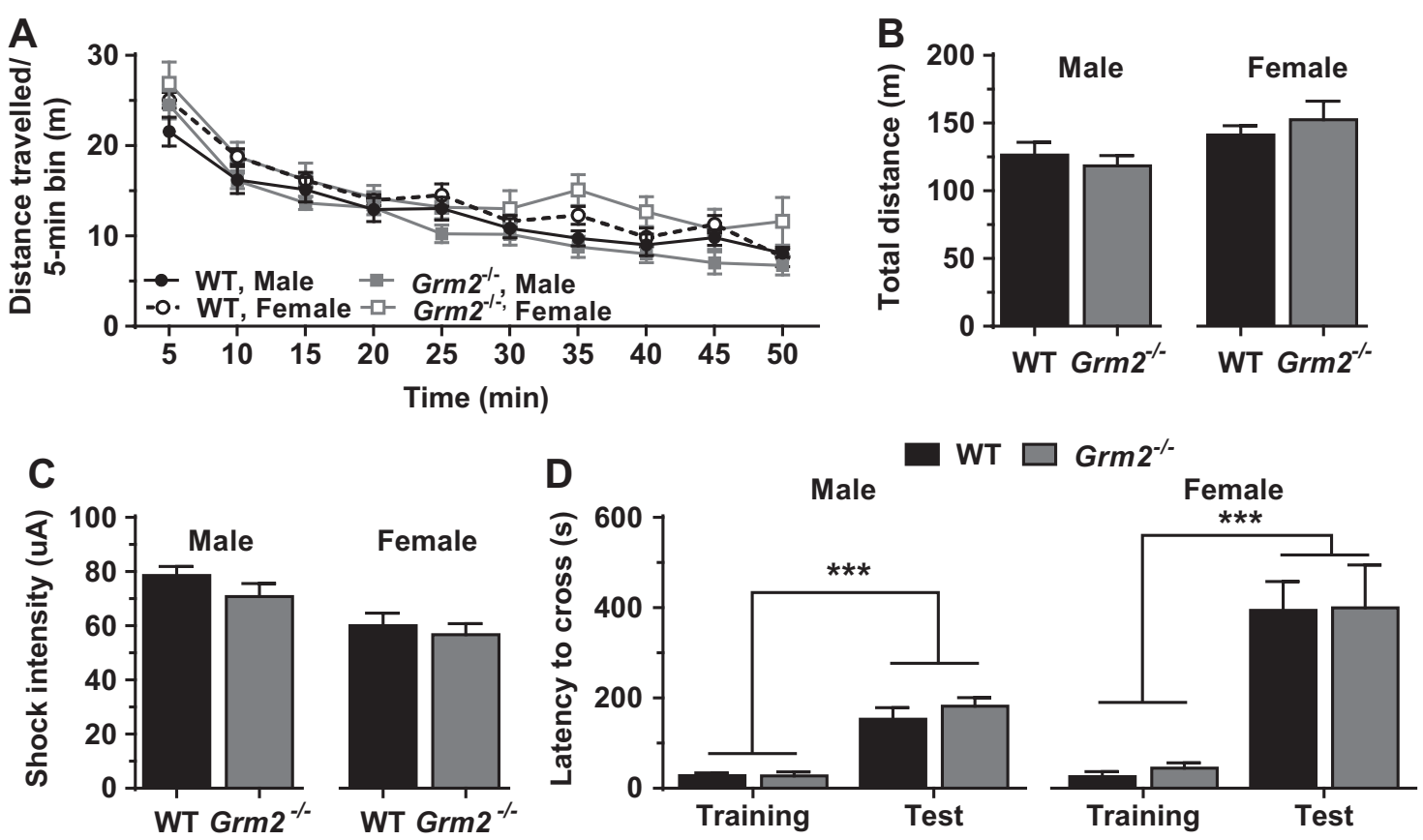

Fig. 3 Mice lacking $\mathrm{mGlu}_{2}$ do not have alterations in locomotor activity, sensitivity to shock, or passive avoidance behavior. Mice lacking $\mathrm{mGlu}_{2}\left(\mathrm{Grm}^{-/-}\right)$do not manifest deficits in $(\mathbf{a}, \mathbf{b})$ open-field locomotor activity (a, distance over time; $\mathbf{b}$, total distance travelled; $n=10-14 /$ sex and genotype), (c) pain sensitivity to shock ( $n=12-14 /$ sex and genotype), or (d) escape latency in the passive avoidance test ( $n=3-5 /$ sex and genotype), compared with wild-type (WT) mice. Independent of genotype, female mice have higher locomotor activity (a, b), lower threshold for shock response (c), and higher escape latency in the passive avoidance test (d) than males. (Data are mean \pm SEM. ${ }^{*} p<0.05,{ }^{* * *} p<0.001$. See Table S1 for reporting of statistical analyses and individual sample sizes

(Fig. 5g, Test 3) of IES-induced escape deficits without altering recovery (Fig. $5 g$, Test 2).

Microinjection of LY341495 into the mPFC recapitulates the effects of systemic administration

To test the involvement of $\mathrm{mGlu}_{2 / 3}$ specifically in the mPFC in mediating the stress-protective effects of LY341495, we evaluated the onset and reinstatement of IES-induced escape deficits following microinjection of LY341495 into the mPFC of mice. Similar to systemic administration, bilateral microinjection of LY341495 (1.0 pmol per side) into the mPFC (placements shown in Fig 5i) 3 days prior to IES (see Fig. $5 \mathrm{~d}$ for timeline) prevented both the initial development (Fig. 5h, Test 1) and reinstatement (Fig. 5h, Test 3) of IES-induced escape deficits, without altering recovery (Fig. 5h, Test 2). While a ten-fold lower dose of LY341495 (0.10 pmol per side) did not prevent the initial bout of escape deficits (Fig. 5h, Test 1) or alter recovery (Fig. 5h, Test 2) from the initial IES, this dose was effective at preventing the reinstatement of this maladaptive behavior (Fig. 5h, Test 3).

\section{DISCUSSION}

In the present study we compared a number of stress-induced behavioral changes in $\mathrm{Grm2}^{-1-}$ and WT mice. Grm2 ${ }^{-1-}$ mice demonstrated stress resilience in all behavioral tests. Namely, $\mathrm{Grm}^{-1-}$ mice manifested lower FST immobility time (reduced behavioral despair) and were more resilient to developing IES- and CORT-induced escape deficits and CSDS-induced anhedonia (sucrose preference deficits), relative to WT controls. Importantly, no alterations in locomotor activity, shock sensitivity, or passive avoidance were observed in $\mathrm{Grm}^{-1-}$ mice. Further, $\mathrm{Grm}^{-1-}$ mice did not manifest altered plasma CORT levels under baseline conditions or following IES, suggesting that their resilient phenotype is not mediated by altered CORT levels. Unlike $\mathrm{Grm}^{-1-}$ mice, $\mathrm{Grm}^{-1-}$ mice were indistinguishable from WT controls in their FST immobility time and susceptibility to IES- induced escape deficits, consistent with a $\mathrm{mGlu}_{2}$ subtype-specific protective effect. These findings are also consistent with previous studies demonstrating that the antidepressant-like behavioral actions of $\mathrm{mGlu}_{2 / 3}$ antagonists are mediated by $\mathrm{mGlu}_{2}$ inhibition $[28,29]$. Although an overall effect of sex was observed for locomotor activity, shock sensitivity, and passive avoidance latency, no effect of sex was observed for any of the stressinduced behavioral outcomes, indicating that $\mathrm{Grm}^{-1-}$ mice exhibit stress resilience, independent of sex.

Our results suggest that selective $\mathrm{mGlu}_{2}$ inhibitors may be effective in inducing stress resilience. However, due to limited commercially available subtype-specific $\mathrm{mGlu}_{2}$ inhibitors, we utilized the group II selective antagonist LY341495 to assess whether pharmacological blockade of $\mathrm{mGlu}_{2 / 3}$ could enhance stress resilience. In addition, to determine whether the effects of $\mathrm{mGlu}_{2 / 3}$ activity on stress resilience were bidirectional, we also evaluated the effects of activation of $\mathrm{mGlu}_{2 / 3}$ by the agonist LY379268. During stress exposure (i.e., treatment $35 \mathrm{~min}$ prior to stress), blockade of $\mathrm{mGlu}_{2 / 3}$ enhanced stress resilience, whereas activation of these receptors increased susceptibility. Pretreatment with LY341495 1, 3, or 7 days prior to IES, prevented the development of maladaptive escape deficits, demonstrating that a single administration results in sustained enhancement of stress resilience and protection from later stressors. Thus, drugs that inhibit $\mathrm{mGlu}_{2}$ activity may be effective prophylactic treatments to prevent the onset of stress-induced mood disorders, including depression, in susceptible populations where exposure to severe stress is anticipated.

Another potential application for stress-enhancing agents is protection against the recurrence of subsequent episodes in depressed patients, a need frequently unmet by current treatments [2]. To evaluate whether treatment with LY341495 could protect against the recurrence of maladaptive behaviors following subsequent stressors, we first established a novel model to recapitulate the recurrent nature of stress exposures leading to depression. Following the initial development of 
A

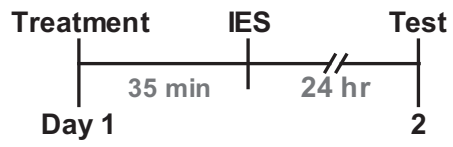

D

G

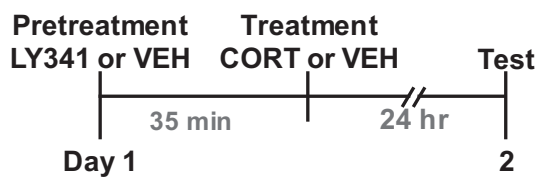

B

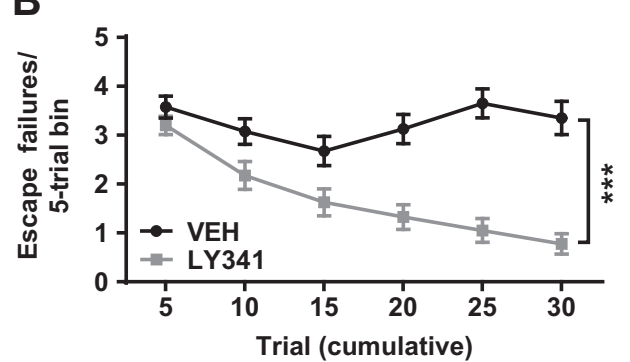

E

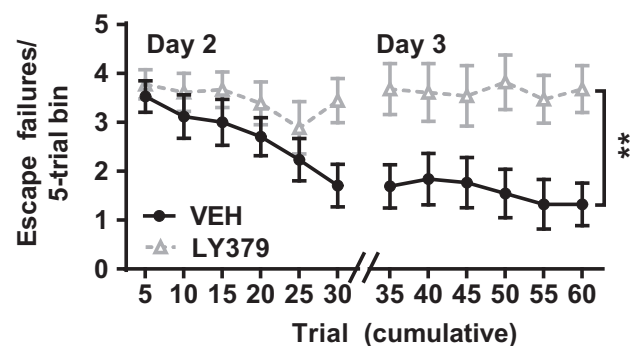

H

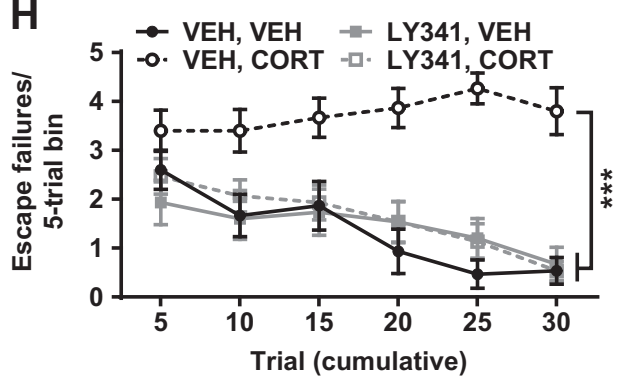

L

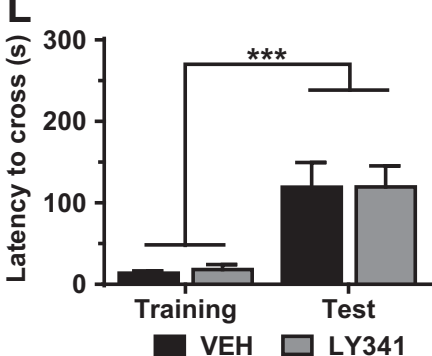

C

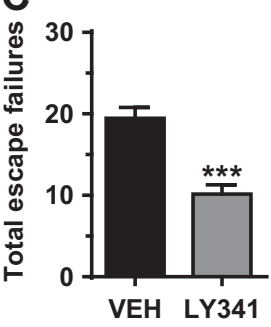

$\mathbf{F}$

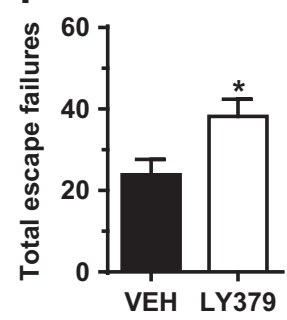

I

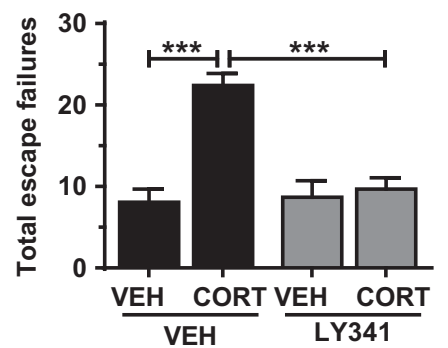

M

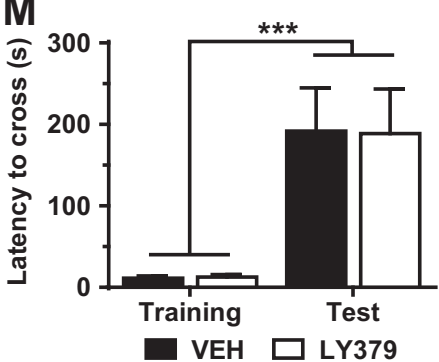

Fig. 4 Pharmacological modulation of $\mathrm{mGlu}_{2 / 3}$ activity during stress modulates resilience versus susceptibility to developing escape deficits. Treatment with the selective $\mathrm{mGlu}_{2 / 3}$ antagonist LY341495 (LY341, $3.0 \mathrm{mg} / \mathrm{kg}$ ) $35 \mathrm{~min}$ prior to (a-c) inescapable shock (IES) stress (see a for timeline; $\mathbf{b}$, failures over time; $\mathbf{c}$, total failures; $n=40 /$ group) or $(\mathbf{g}-\mathbf{i})$ acute corticosterone (CORT, $10 \mathrm{mg} / \mathrm{kg}$ ) treatment (see $\mathbf{g}$ for timeline; $\mathbf{h}$, failures over time; $\mathbf{i}$, total failures $n=15$ /group) prevented the development of escape deficits (i.e., enhanced resilience). Treatment with the selective agonist LY379268 (LY379, $3.0 \mathrm{mg} / \mathrm{kg})$ at the same time point relative to IES (d-f) increased escape deficits (i.e., increased susceptibility) in a modified 3-day test (see $\mathbf{d}$ for timeline; e, failures over time; $\mathbf{f}$, total failures; $n=17-18 /$ group). Neither treatment altered (j, k) pain sensitivity to shock (see $\mathbf{j}$ for timeline; $n=10 /$ group) nor $\mathbf{( j ,}, \mathbf{l}, \mathbf{m})$ performance in the passive avoidance test (see $\mathbf{j}$ for timeline; $n=9-10$ / group). Data are mean \pm SEM. ${ }^{*} p<0.05,{ }^{* *} p<0.01,{ }^{* * *} p<0.001$. See Table S1 for reporting of statistical analyses and individual sample sizes

IES-induced escape deficits, mice recovered within 2 weeks (12 days after IES), no longer displaying the maladaptive behavior. Subsequent exposure to an abbreviated IES session (six times shorter than the full-length session), however, reinstated maladaptive escape deficits in vehicle-treated mice. In the same model, prophylactic treatment with LY341495 3 or 7 days prior to the initial IES session was protective against not only the initial bout of escape deficits, but also the reinstatement of this maladaptive behavior by re-exposure to IES 16 or 20 days after treatment. These results demonstrate long-lasting protective effects of a single systemic treatment with LY341495, and suggest that $\mathrm{mGlu}_{2 / 3}$ inhibitors may be effective in preventing the recurrence of stress-related depressive episodes.
While previous work has demonstrated that, in rodents, $\mathrm{mGlu}_{2 / 3}$ antagonists exert antidepressant-like effects, or reverse previously established maladaptive behaviors [16-28], our data extend the potential applications of $\mathrm{mGlu}_{2 / 3}$ antagonists from reversal to prevention. Here we demonstrate, for the first time, that a single treatment with an $\mathrm{mGlu}_{2 / 3}$ antagonist induces prolonged stress resilience (lasting up to 20 days), protecting against both the initial onset and recurrence of stress-induced behavioral deficits. Although several interventions, including environmental enrichment $[37,38]$ and voluntary wheel-running (reviewed in [39]), have been reported to increase stress resilience in rodents, pharmacological approaches to enhance stress resilience have not been extensively studied. Recently, however, the rapid-acting antidepressant ketamine has been 
A

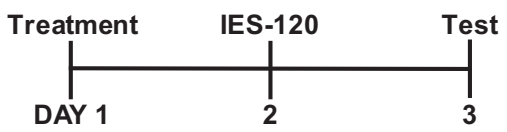

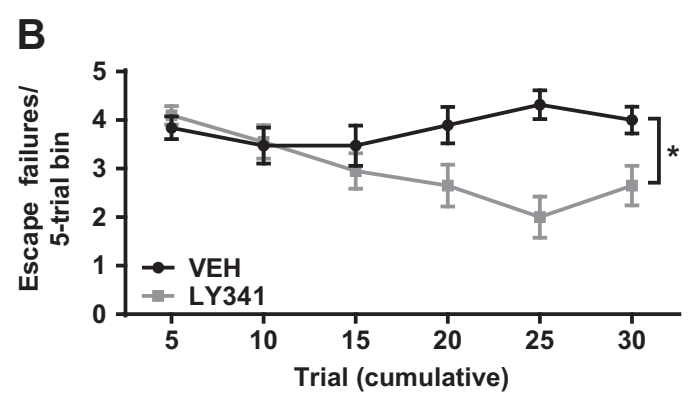

C

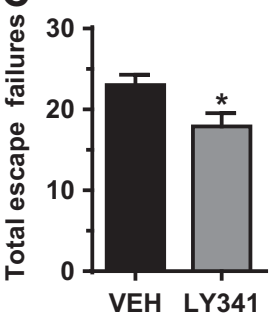

D

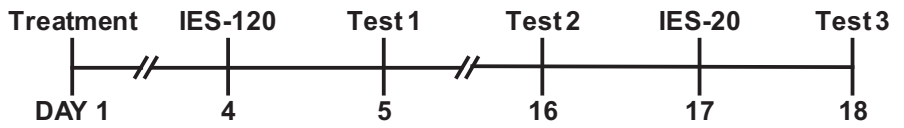

\section{E}

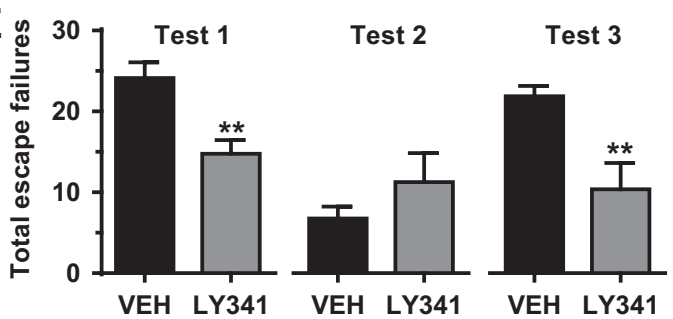

F

G
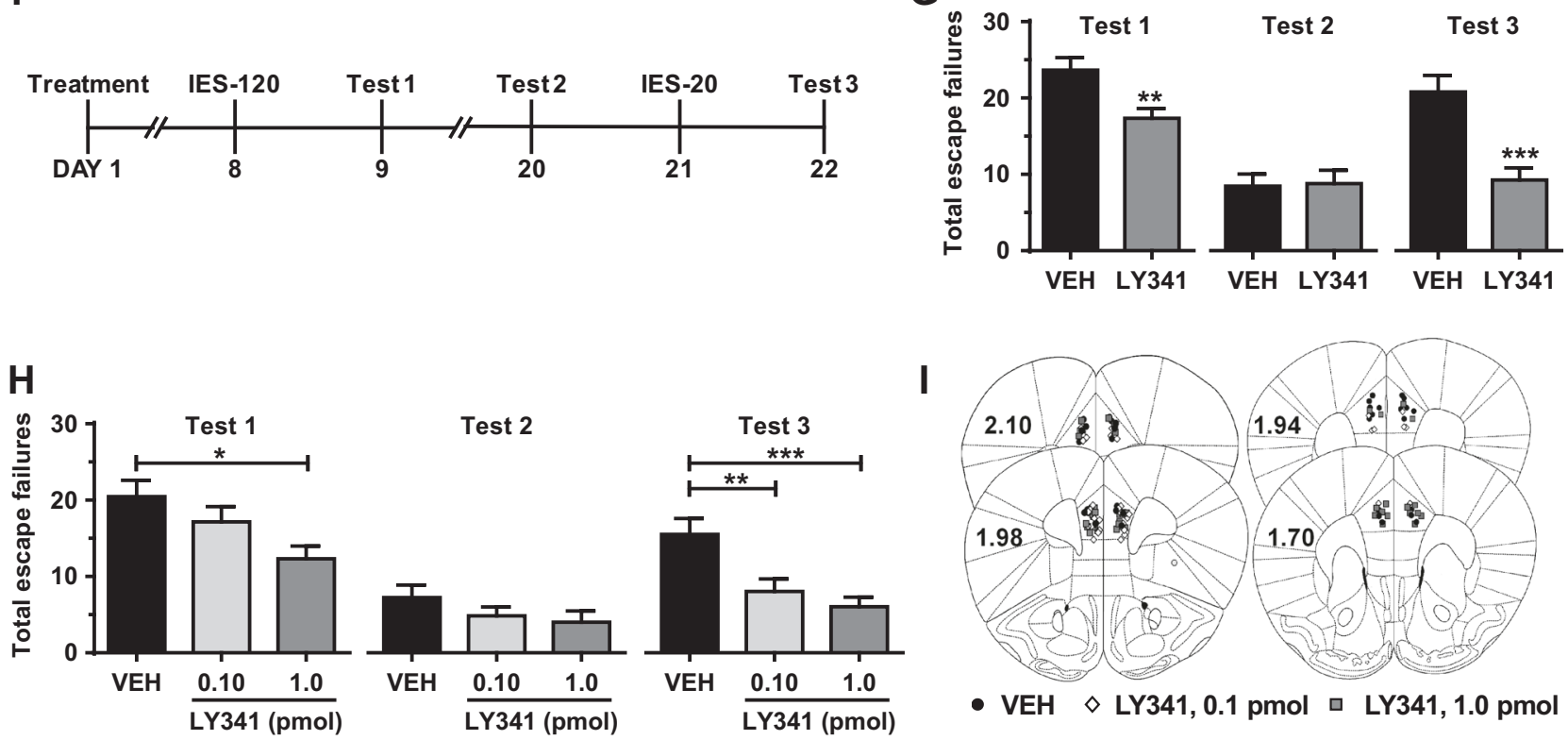

Fig. 5 Pharmacological blockade of $\mathrm{mGlu}_{2 / 3}$ prior to stress exposure prevents the development and recurrence shock-induced escape deficits. (a-c) Treatment with the $\mathrm{mGlu}_{2 / 3}$ antagonist LY341495 (LY341, $3.0 \mathrm{mg} / \mathrm{kg}$ ) $24 \mathrm{~h}$ before inescapable shock (IES) stress (see a for timeline) prevents the development of (b, c) IES-induced escape deficits (b, failures over time; $\mathbf{c}$, total failures; $n=19-20 /$ group). In a repeated IES stress paradigm (see d for timeline), treatment with LY341495 3 days before the initial IES session (120 shocks; IES-120) prevents (e, Test 1) the initial development of escape deficits and (e, Test 3 ) the recurrence of escape deficits following exposure to a subthreshold IES session (20 shocks; IES-20), without altering (e, Test 2) recovery ( $n=8$ /group). In the same paradigm, treatment with LY341495 7 days before the initial IES session (see $\mathbf{f}$ for timeline) prevents (g, Test 1) the development and (g, Test 3) recurrence of escape deficits, without altering (g, Test 2) recovery ( $n=15 /$ group). Microinjection of LY341495 into the MPFC (see i for cannula placements) 3 days before IES, recapitulates the effects of systemic treatment, preventing both the (h, Test 1) initial onset and (h, Test 3) reinstatement of escape deficits, without altering (h, Test 2$)$ recovery ( $n=18 /$ group). Data are mean \pm SEM. ${ }^{*} p<0.05,{ }^{* *} p<0.01$. See Table S1 for reporting of statistical analyses and individual sample sizes

recognized for its ability to prevent deleterious effects of stress in rodent studies [40-43]. Notably, ketamine has been suggested to share convergent mechanisms of antidepressant action with $\mathrm{mGlu}_{2 / 3}$ antagonists (see [44]), both involving an increase in glutamate release and excitatory synapse potentiation [6, 27, 44], a-amino-3-hydroxy-5-methyl-4-isoxazolepropionic acid receptor (AMPAR) activation [24, 45, 46], and downstream activation of mammalian target of rapamycin (mTOR) and synthesis of synaptic proteins including BDNF [5, $17,18,23,47]$. Thus, it is possible that the stress-protective effects of these two compounds share similar overlapping mechanisms.
While the full mechanisms have not been elucidated, it has been proposed that pretreatment with ketamine protects against subsequent stressors by increasing glutamatergic activity in the mPFC and downstream activation of glutamatergic projections from the mPFC to the DRN, a neural circuit whose role in stress resilience has been extensively studied (reviewed in [48]). Activation of these glutamatergic projections, which synapse preferentially onto gamma-aminobutyric acid (GABA)-ergic interneurons in the DRN, has an inhibitory effect on serotonergic signaling in the DRN and its projections [48, 49]. Excessive activation of these serotonergic pathways is involved in the development of uncontrollable stress-induced behavioral deficits, 
including those induced by IES in rodents $[8,49,50]$. Thus, manipulations that activate these glutamatergic mPFC to DRN projections, including ketamine, prevent this stress-induced aberrant serotonergic activation and its behavioral consequences (i.e., confer stress resilience) $[8,37,42,50]$. Systemic administration of LY341495 has been reported to increase c-Fos labeling in prelimbic areas [51], consistent with a site of action that includes the PFC. We hypothesized that blockade of $\mathrm{mGlu}_{2 / 3}$ in the $\mathrm{mPFC}$, similar to ketamine, would enhance stress resilience. Consistent with this hypothesis, microinjection of LY341495 into the MPFC 3 days prior to IES prevented the development of IES-induced escape deficits and, moreover, was protective against the recurrence of such deficits after a recovery period. Interestingly, even when injected into the MPFC at a dose that did not prevent the initial bout of escape deficits $(0.10 \mathrm{pmol} / \mathrm{side})$, LY341495 protected against the reinstatement of this behavior by a subsequent stress exposure. This result suggests that lower doses of LY341495 may be sufficient to induce the synaptic changes that provide continued protection against recurrence of stress-induced depressive-like episodes, compared with those required to prevent the initial onset of these behaviors. Overall, these data implicate the mPFC as a critical site of action for the lasting stressprotective actions of LY341495, and are consistent with previous reports that microinjection of LY341495 into the MPFC exerts antidepressant-like effects [25] which is consistent with overlapping mechanisms underlying the prevention and reversal of maladaptive stress-induced changes. However, further studies are necessary to confirm the involvement of the MPFC to DRN circuit in mediating its stress-protective actions and to elucidate the precise mechanisms underlying these effects.

Contrary to our findings and those of other laboratories [16-28], there have been reports that increased $\mathrm{mGlu}_{2}$ expression or activity induces stress resilience or antidepressant-relevant effects. Namely, L-acetylcarnitine, which epigenetically upregulates $\mathrm{mGlu}_{2}$ expression, has been reported to exert antidepressant-like effects in rodents [52], and the same group has reported that $\mathrm{Grm} 2^{-/-}$ mice (C57BL/6 background) exhibit increased stress susceptibility following chronic unpredictable stress [53]. It is unclear whether differences in the experimental animal strain, specific stress paradigms used, or brain regions affected by individual treatments (i.e., L-acetylcarnitine vs. LY341495) contribute to these seemingly paradoxical effects of $\mathrm{mGlu}_{2 / 3}$ modulation, and further studies are required to understand the complete involvement of $\mathrm{mGlu}_{2}$ activity in stress-related disorders including depression.

Nonetheless, we demonstrate that prophylactic blockade of $\mathrm{mGlu}_{2 / 3}$ induces lasting stress resilience. Our data reveal, for the first time, that prophylactic treatment with an $\mathrm{mGlu}_{2 / 3}$ antagonist is protective not only against the initial onset of stress-induced behavioral deficits up to 7 days after treatment, but also against the recurrence of such deficits by subsequent stressors up to 20 days after treatment. In addition, our studies reveal that subtype-specific deletion of $\mathrm{mGlu}_{2}$, but not $\mathrm{mGlu}_{3}$, confers a stress-resilient phenotype in mice, consistent with the stressprotective effects of LY341495 being mediated by $\mathrm{mGlu}_{2}$ inhibition. Overall, our data predict that agents that negatively modulate $\mathrm{mGlu}_{2}$ activity may be effective therapies to prevent the onset and/or recurrence of stress-related disorders such as depression.

\section{FUNDING AND DISCLOSURE}

This work was supported by $\mathrm{NIH}$ grant $\mathrm{MH} 107615$ and a Harrington Discovery Institute Scholar-Innovator grant to T.D.G. and NIGMS T32 training grant GM008181 to J.N.H. The University of Virginia Center for Research in Reproduction Ligand Assay and Analysis Core is supported by the Eunice Kennedy Shriver NICHD/ $\mathrm{NIH}$ (NCTRI) Grant P50-HD28934. The contents do not represent the views of the U.S. Department of Veterans Affairs or the United
States Government. T.D.G. has received research funding from Janssen, Allergan, and Roche Pharmaceuticals, and a consultant for FSV7 LLC, during the preceding 3 years. The authors declare no competing interests.

\section{ADDITIONAL INFORMATION}

Supplementary Information this paper this paper at (https://doi.org/10.1038/ s41386-019-0380-1).

Publisher's note: Springer Nature remains neutral with regard to jurisdictional claims in published maps and institutional affiliations.

\section{REFERENCES}

1. Rush AJ, Trivedi MH, Wisniewski SR, Nierenberg AA, Stewart JW, Warden D, et al. Acute and longer-term outcomes in depressed outpatients requiring one or several treatment steps: a STAR*D report. Am J Psychiatry. 2006;163:1905-17.

2. Hardeveld F, Spijker J, De Graaf R, Nolen WA, Beekman AT. Prevalence and predictors of recurrence of major depressive disorder in the adult population. Acta Psychiatr Scand. 2010;122:184-91.

3. Burcusa SL, lacono WG. Risk for recurrence in depression. Clin Psychol Rev. 2007:27:959-85.

4. Kessler RC. The effects of stressful life events on depression. Annu Rev Psychol. 1997;48:191-214.

5. Wohleb ES, Gerhard D, Thomas A, Duman RS. Molecular and cellular mechanisms of rapid-acting antidepressants ketamine and scopolamine. Curr Neuropharmacol. 2017;15:11-20.

6. Thompson SM, Kallarackal AJ, Kvarta MD, Van Dyke AM, LeGates TA, Cai X. An excitatory synapse hypothesis of depression. Trends Neurosci. 2015;38:279-94.

7. Kallarackal AJ, Kvarta MD, Cammarata E, Jaberi L, Cai X, Bailey AM, et al. Chronic stress induces a selective decrease in AMPA receptor-mediated synaptic excitation at hippocampal temporoammonic-CA1 synapses. J Neurosci. 2013; 33:15669-74.

8. Amat J, Paul E, Watkins LR, Maier SF. Activation of the ventral medial prefrontal cortex during an uncontrollable stressor reproduces both the immediate and long-term protective effects of behavioral control. Neuroscience. 2008;154:1178-86.

9. Conn PJ, Pin JP. Pharmacology and functions of metabotropic glutamate receptors. Annu Rev Pharmacol Toxicol. 1997;37:205-37.

10. Kiritoshi T, Neugebauer V. Group II mGluRs modulate baseline and arthritis painrelated synaptic transmission in the rat medial prefrontal cortex. Neuropharmacology. 2015;95:388-94.

11. Hascup ER, Hascup KN, Stephens M, Pomerleau F, Huettl P, Gratton A, et al. Rapid microelectrode measurements and the origin and regulation of extracellular glutamate in rat prefrontal cortex. J Neurochem. 2010;115:1608-20.

12. Shigemoto R, Kinoshita A, Wada E, Nomura S, Ohishi H, Takada M, et al. Differential presynaptic localization of metabotropic glutamate receptor subtypes in the rat hippocampus. J Neurosci. 1997;17:7503-22.

13. Schoepp DD. Unveiling the functions of presynaptic metabotropic glutamate receptors in the central nervous system. J Pharmacol Exp Ther. 2001 299:12-20.

14. Joffe ME, Conn PJ. Antidepressant potential of metabotropic glutamate receptor mGlu2 and mGlu3 negative allosteric modulators. Neuropsychopharmacology. 2018;44:214-36.

15. Ohishi $H$, Shigemoto $R$, Nakanishi S, Mizuno N. Distribution of the mRNA for a metabotropic glutamate receptor (mGluR3) in the rat brain: an in situ hybridization study. J Comp Neurol. 1993;335:252-66.

16. Chaki S, Yoshikawa R, Hirota S, Shimazaki T, Maeda M, Kawashima N, et al. MGS0039: a potent and selective group II metabotropic glutamate receptor antagonist with antidepressant-like activity. Neuropharmacology. 2004; 46:457-67.

17. Dwyer JM, Lepack $A E$, Duman RS. mTOR activation is required for the antidepressant effects of $\mathrm{mGluR}(2) /(3)$ blockade. Int J Neuropsychopharmacol. 2012;15:429-34.

18. Koike $H$, Fukumoto $K$, lijima $M$, Chaki $S$. Role of BDNF/TrkB signaling in antidepressant-like effects of a group II metabotropic glutamate receptor antagonist in animal models of depression. Behav Brain Res. 2013;238:48-52.

19. Dwyer JM, Lepack AE, Duman RS. mGluR2/3 blockade produces rapid and longlasting reversal of anhedonia caused by chronic stress exposure. J Mol Psychiatry. 2013;1:15.

20. Ago Y, Yano K, Araki R, Hiramatsu N, Kita Y, Kawasaki T, et al. Metabotropic glutamate $2 / 3$ receptor antagonists improve behavioral and prefrontal 
dopaminergic alterations in the chronic corticosterone-induced depression model in mice. Neuropharmacology. 2013;65:29-38.

21. Campo B, Kalinichev M, Lambeng N, El Yacoubi M, Royer-Urios I, Schneider M, et al. Characterization of an mGluR2/3 negative allosteric modulator in rodent models of depression. J Neurogenet. 2011;25:152-66.

22. Dong C, Zhang JC, Yao W, Ren Q, Ma M, Yang C, et al. Rapid and sustained antidepressant action of the $\mathrm{mGlu2/3}$ receptor antagonist MGS0039 in the social defeat stress model: comparison with ketamine. Int J Neuropsychopharmacol. 2017;20:228-36.

23. Koike $\mathrm{H}$, lijima $\mathrm{M}$, Chaki S. Involvement of the mammalian target of rapamycin signaling in the antidepressant-like effect of group II metabotropic glutamate receptor antagonists. Neuropharmacology. 2011;61:1419-23.

24. Koike $\mathrm{H}$, Chaki $\mathrm{S}$. Requirement of AMPA receptor stimulation for the sustained antidepressant activity of ketamine and LY341495 during the forced swim test in rats. Behav Brain Res. 2014;271:111-5.

25. Fukumoto K, lijima M, Chaki S. The antidepressant effects of an mGlu2/3 receptor antagonist and ketamine require AMPA receptor stimulation in the MPFC and Subsequent activation of the 5-HT neurons in the DRN. Neuropsychopharmacology. 2016;41:1046-56.

26. Witkin JM, Marek GJ, Johnson BG, Schoepp DD. Metabotropic glutamate receptors in the control of mood disorders. CNS Neurol Disord Drug Targets. 2007;6:87-100.

27. Chaki S. mGlu2/3 receptor antagonists as novel antidepressants. Trends Pharmacol Sci. 2017:38:569-80.

28. Witkin JM, Mitchell SN, Wafford KA, Carter G, Gilmour G, Li J, et al. Comparative effects of LY3020371, a potent and selective metabotropic glutamate (mGlu) 2/3 receptor antagonist, and ketamine, a noncompetitive $\mathrm{N}$-methyl-d-aspartate receptor antagonist in rodents: evidence supporting the use of mGlu2/3 antagonists, for the treatment of depression. J Pharmacol Exp Ther. 2017;361:68-86.

29. Gleason SD, Li X, Smith IA, Ephlin JD, Wang XS, Heinz BA, et al. mGlu2/3 agonistinduced hyperthermia: an in vivo assay for detection of $\mathrm{mGlu2} / 3$ receptor antagonism and its relation to antidepressant-like efficacy in mice. CNS Neurol Disord Drug Targets. 2013;12:554-66.

30. Ornstein PL, Bleisch TJ, Arnold MB, Kennedy JH, Wright RA, Johnson BG, et al. 2substituted (2SR)-2-amino-2-((1SR,2SR)-2-carboxycycloprop-1-yl)glycines as potent and selective antagonists of group II metabotropic glutamate receptors. 2 . Effects of aromatic substitution, pharmacological characterization, and bioavailability. J Med Chem. 1998;41:358-78.

31. Linden AM, Shannon H, Baez M, Yu JL, Koester A, Schoepp DD. Anxiolytic-like activity of the mGLU2/3 receptor agonist LY354740 in the elevated plus maze test is disrupted in metabotropic glutamate receptor 2 and 3 knock-out mice. Psychopharmacology (Berl). 2005;179:284-91.

32. Wright RA, Johnson BG, Zhang C, Salhoff $C$, Kingston AE, Calligaro DO, et al. CNS distribution of metabotropic glutamate 2 and 3 receptors: transgenic mice and [(3)H]LY459477 autoradiography. Neuropharmacology. 2013;66:89-98.

33. Hajszan T, Dow A, Warner-Schmidt JL, Szigeti-Buck K, Sallam NL, Parducz A, et al. Remodeling of hippocampal spine synapses in the rat learned helplessness model of depression. Biol Psychiatry. 2009;65:392-400.

34. Carter BS, Hamilton DE, Thompson RC. Acute and chronic glucocorticoid treatments regulate astrocyte-enriched mRNAs in multiple brain regions in vivo. Front Neurosci. 2013;7:139.

35. Krishnan V, Han MH, Graham DL, Berton O, Renthal W, Russo SJ, et al. Molecular adaptations underlying susceptibility and resistance to social defeat in brain reward regions. Cell. 2007;131:391-404.
36. Golden SA, Covington HE III, Berton O, Russo SJ. A standardized protocol for repeated social defeat stress in mice. Nat Protoc. 2011;6:1183-91.

37. Lehmann ML, Herkenham M. Environmental enrichment confers stress resiliency to social defeat through an infralimbic cortex-dependent neuroanatomical pathway. J Neurosci. 2011;31:6159-73.

38. Ashokan A, Hegde A, Mitra R. Short-term environmental enrichment is sufficient to counter stress-induced anxiety and associated structural and molecular plasticity in basolateral amygdala. Psychoneuroendocrinology. 2016;69:189-96.

39. Greenwood BN, Fleshner M. Exercise, learned helplessness, and the stressresistant brain. Neuromolecular Med. 2008;10:81-98.

40. Brachman RA, McGowan JC, Perusini JN, Lim SC, Pham TH, Faye C, et al. Ketamine as a prophylactic against stress-induced depressive-like behavior. Biol Psychiatry. 2016;79:776-86.

41. McGowan JC, LaGamma CT, Lim SC, Tsitsiklis M, Neria Y, Brachman RA, et al. Prophylactic ketamine attenuates learned fear. Neuropsychopharmacology. 2017:42:1577-89.

42. Amat J, Dolzani SD, Tilden S, Christianson JP, Kubala KH, Bartholomay K, et al. Previous ketamine produces an enduring blockade of neurochemical and behavioral effects of uncontrollable stress. J Neurosci. 2016;36:153-61.

43. Mastrodonato A, Martinez R, Pavlova IP, LaGamma CT, Brachman RA, Robison AJ, et al. Ventral CA3 activation mediates prophylactic ketamine efficacy against stress-induced depressive-like behavior. Biol Psychiatry. 2018;84:846-56.

44. Zanos P, Thompson SM, Duman RS, Zarate CA Jr., Gould TD. Convergent mechanisms underlying rapid antidepressant action. CNS Drugs. 2018; 32:197-227.

45. Witkin JM, Monn JA, Schoepp DD, Li X, Overshiner C, Mitchell SN, et al. The rapidly acting antidepressant ketamine and the mGlu2/3 receptor antagonist LY341495 rapidly engage dopaminergic mood circuits. J Pharmacol Exp Ther. 2016;358:71-82.

46. Palucha-Poniewiera A, Wieronska JM, Branski P, Stachowicz K, Chaki S, Pilc A. On the mechanism of the antidepressant-like action of group II mGlu receptor antagonist, MGS0039. Psychopharmacology (Berl). 2010;212:523-35. PMCID: 2981731

47. Autry $A E$, Adachi $M$, Nosyreva $E, N a E S$, Los MF, Cheng PF, et al. NMDA receptor blockade at rest triggers rapid behavioural antidepressant responses. Nature. 2011:475:91-5.

48. Maier SF, Seligman ME. Learned helplessness at fifty: insights from neuroscience. Psychol Rev. 2016;123:349-67.

49. Maier SF, Watkins LR. Stressor controllability and learned helplessness: the roles of the dorsal raphe nucleus, serotonin, and corticotropin-releasing factor. Neurosci Biobehav Rev. 2005;29:829-41.

50. Amat J, Aleksejev RM, Paul E, Watkins LR, Maier SF. Behavioral control over shock blocks behavioral and neurochemical effects of later social defeat. Neuroscience. 2010;165:1031-8.

51. Linden AM, Bergeron M, Schoepp DD. Comparison of c-Fos induction in the brain by the mGlu2/3 receptor antagonist LY341495 and agonist LY354740: evidence for widespread endogenous tone at brain mGlu2/3 receptors in vivo. Neuropharmacology. 2005;49:120-34.

52. Nasca C, Xenos D, Barone Y, Caruso A, Scaccianoce S, Matrisciano F, et al. Lacetylcarnitine causes rapid antidepressant effects through the epigenetic induction of mGlu2 receptors. Proc Natl Acad Sci USA. 2013;110:4804-9.

53. Nasca C, Bigio B, Zelli D, Nicoletti F, McEwen BS. Mind the gap: glucocorticoids modulate hippocampal glutamate tone underlying individual differences in stress susceptibility. Mol Psychiatry. 2015;20:755-63. 